\title{
Initial hospital management of patients with emergent large vessel occlusion (ELVO): report of the standards and guidelines committee of the Society of Neurolnterventional Surgery
}

\author{
Ryan A McTaggart, ${ }^{1}$ Sameer A Ansari, ${ }^{2}$ Mayank Goyal, ${ }^{3}$ Todd A Abruzzo, ${ }^{4}$ \\ Barb Albani, ${ }^{5}$ Adam J Arthur, ${ }^{6}$ Michael J Alexander, ${ }^{7}$ Felipe C Albuquerque, ${ }^{8}$ \\ Blaise Baxter, ${ }^{9}$ Ketan R Bulsara, ${ }_{10}$ Michael Chen, ${ }^{11}$ Josser E Delgado Almandoz, ${ }^{12}$ \\ Justin F Fraser, ${ }^{13}$ Donald Frei, ${ }^{14}$ Chirag D Gandhi, ${ }^{15}$ Don V Heck, ${ }^{16}$ Steven W Hetts, ${ }^{17}$ \\ M Shazam Hussain, ${ }^{18}$ Michael Kelly, ${ }^{19}$ Richard Klucznik, ${ }^{20}$ Seon-Kyu Lee, ${ }^{21}$ \\ Thabele Leslie-Mawzi, ${ }^{22}$ Philip M Meyers, ${ }^{23}$ Charles J Prestigiacomo, ${ }^{15} \mathrm{G}$ Lee Pride, ${ }^{24}$ \\ Athos Patsalides, ${ }^{25}$ Robert M Starke, ${ }^{26}$ Peter Sunenshine, ${ }^{27}$ Peter A Rasmussen, ${ }^{18}$ \\ Mahesh V Jayaraman, ${ }^{1}$ on behalf of the Standards and Guidelines Committee of the \\ Society of Neurolnterventional Surgery (SNIS)
}

For numbered affiliations see end of article.

\section{Correspondence to}

Dr Ryan A McTaggart, Warren Alpert Medical School of Brown University, Providence, RI 02903, USA;

ryan.mctaggart@lifespan.org

Received 27 July 2015 Accepted 31 July 2015 Published Online First 31 August 2015

\section{CrossMark}

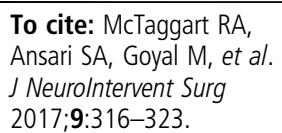

\section{ABSTRACT}

Objective To summarize the current literature regarding the initial hospital management of patients with acute ischemic stroke (AIS) secondary to emergent large vessel occlusion (ELVO), and to offer recommendations designed to decrease the time to endovascular treatment (EVT) for appropriately selected patients with stroke.

Methods Using guidelines for evidenced-based medicine proposed by the Stroke Council of the American Heart Association, a critical review of all available medical literature supporting best initial medical management of patients with AIS secondary to ELVO was performed. The purpose was to identify processes of care that most expeditiously determine the eligibility of a patient with an acute stroke for interventions including intravenous fibrinolysis with recombinant tissue plasminogen activator (IV TPA) and EVT using mechanical embolectomy.

Results This review identifies four elements that are required to achieve timely revascularization in ELVO. (1) In addition to non-contrast CT (NCCT) brain scan, CT angiography should be performed in all patients who meet an institutional threshold for clinical stroke severity. The use of any advanced imaging beyond NCCT should not delay the administration of IV IPA in eligible patients. (2) Activation of the neurointerventional team should occur as soon as possible, based on either confirmation of large vessel occlusion or a prespecified clinical severity threshold. (3) Additional imaging techniques, particularly those intended to physiologically select patients for EVT (CT perfusion and diffusionperfusion mismatch imaging), may provide additional value, but should not delay EVT. (4) Routine use of general anesthesia during EVT procedures, should be avoided if possible. These workflow recommendations apply to both primary and comprehensive stroke centers and should be tailored to meet the needs of individual institutions.

Conclusions Patients with ELVO are at risk for severe neurologic morbidity and mortality. To achieve the best possible clinical outcomes stroke centers must optimize their triage strategies. Strategies that provide patients with ELVO with the fastest access to reperfusion depend upon detail-oriented process improvement.

\section{INTRODUCTION}

Within the past year, clinical trials have established American Heart Association (AHA) Class 1 Level A Evidence demonstrating the clinical benefit of modern endovascular therapy, ${ }^{1-5}$ in addition to best medical therapy for patients with acute ischemic stroke (AIS) presenting with emergent large vessel occlusion (ELVO). These studies reiterate that time to revascularization remains an important factor in any effort to achieve good clinical outcomes. Hence, it is now imperative to focus on innovations in the delivery of care and process improvements to create rapid access to both therapies, each critically time dependent. ${ }^{6-8}$ Secondary analysis of the MR CLEAN trial shows that the benefit of revascularization decreases non-linearly with time. ${ }^{8}$ It is important to remember that the effect of time reported in this trial is a summation of all patient outcomes, and the decline in the likelihood of a good outcome for any individual patient may be even more profound. Therefore, the goal of intervention is to achieve revascularization as quickly as possible for the benefit of the patient.

\section{DEFINITIONS/ABBREVIATIONS}

$A I S$ - acute ischemic stroke

ASPECTS - Alberta Stroke Program Early Computed Tomographic Scoring

BRISK - Brisk Recanalization Ischemic Stroke Kit CPSS - Cincinnati Pre-hospital Stroke Scale

Clinical Penumbra - term that implies a discordance between the clinical severity of a stroke and the volume of irreversibly injured brain (infarct core)

CSC - comprehensive stroke center 
CTA - CT angiography

CTP - CT Perfusion

Delivery Innovation - efforts made improve the quality and efficiency of care delivery

Door to CT time - time interval between patient arrival at PSC or CSC and CT acquisition

Door to Needle Time - time interval between patient arrival at PSC or CSC and IV tPA bolus

Door to puncture time - time interval between patient arrival at PSC or CSC and groin access for embolectomy at CSC

DTR - Door to first recanalization - time interval between patient arrival at PSC or CSC and restoration of antegrade flow in the occluded blood vessel at CSC (usually time of stentretriever deployment)

Door to final angiographic run - time interval between patient arrival at PSC or CSC and final angiographic run at CSC at which time final reperfusion score (mTICI) is assigned

DWI - diffusion-weighted imaging

ELVO - Emergent Large Vessel Occlusion

EMS - Emergency Medical Services

ER- Emergency Room

EVT - Endovascular Therapy (such as embolectomy)

Futile infarct - an amount of irreversibly injured brain thought to be incompatible with a favorable or good outcome (see MALCOM)

FAST - Face, arm, speech test

$G A$ - general anesthesia (general endotracheal anesthesia (GETA) also used)

Ischemic Penumbra - oligemic tissue at risk for infarction if rapid revascularization is not achieved

LAMS - Los Angeles Motor Score

$L S W$ - last seen well (does not imply time of onset)

LVO - large vessel occlusion (typically M1 segment and proximal); some authors may include M1 equivalent (2 M2 segment occlusions)

$M A C$ - monitored anesthesia care

$M A L C O M$ - maximal admission lesion volume compatible with favorable outcome

NIHSS - National Institute of Health Stroke Scale

NCCT - Non-contrast CT scan

NNT - number needed to treat (1/absolute difference *100)

Onset to treatment time - time interval from stroke onset to treatment (particular treatment requires specification)

Onset time - when patient last awake and symptom free or known to be "normal"

Picture to puncture - time interval from non-invasive imaging confirmation of ELVO (also known as the qualifying image) to groin puncture

PSC - primary stroke center (i.e. one where EVT is generally not available)

PSC picture to CSC puncture - see picture to puncture above; an important PSC:CSC pair stroke target for performance review $P W I$ - perfusion-weighted imaging (refers to MRI exclusively)

Qualifying Image - this is the image that demonstrates clot occluding blood flow and deemed to potentially require embolectomy

Recanalization - reperfusion is achieved by recanalization (reopening the blood vessel) but recanalization does not always guarantee reperfusion

Reperfusion - preferred term to describe antegrade flow restoration to a cerebral vascular territory. Reperfusion generally implies recanalization was achieved. The degree of reperfusion is most commonly categorized, at present, according to the mTICI score (see below)
$R A C E$ - Rapid Arterial oCclusion Evaluation

ROSIER - Recognition of Stroke in the Emergency Room

Stroke of unknown onset - category of strokes in which the time of onset is unknown thus complicating time threshold treatment decisions (includes wake-up strokes)

Time of onset - the time a stroke started (as distinct from wakeup and LSW)

mTICI - this denotes the modified Thrombolysis in Cerebral

Infarction perfusion scale

mTICI 0 - no antegrade flow

mTICI 1 - contrast material passes beyond the area of occlusion but does not opacify the cerebral bed distal to the occlusive lesion

mTICI $2 \mathrm{a}-<50 \%$ opacification of the cerebral vascular bed beyond the lesion

mTICI $2 b->50 \%$ opacification of the cerebral vascular bed beyond the lesion

mTICI 3 - complete reperfusion

Wake-up stroke - one category of stroke with an unknown time of onset

\section{MATERIALS AND METHODS}

This document was prepared by the Standards and Guidelines Committee of the Society of NeuroInterventional Surgery (SNIS), a multidisciplinary society representing leaders in the field of endovascular therapy for neurovascular disease. The strength of the evidence supporting each recommendation was summarized using a scale previously described by the AHA. In cases where data were insufficient, either no recommendation was made or a recommendation was made based on the consensus of expert practice experience. Other aspects of this care continuum, such as prehospital management, the role of endovascular treatment (EVT), ${ }^{9}$ and post-procedural care, are dealt with separately.

\section{Discussion and recommendations}

For the patient with potential ELVO, we believe there are five initial assessment/management goals before endovascular reperfusion therapy:

1. Confirm an AIS (ie, exclude hemorrhage).

2. Determine candidacy for IV tissue plasminogen activator (tPA) and rapidly administer to all eligible patients.

3. Confirm or exclude the presence of a large vessel occlusion.

4. Determine candidacy for embolectomy, and then activate the neurointerventional team (if at a comprehensive stroke center (CSC)), or transfer to a CSC (if patient is at a primary stroke center (PSC)).

5. Provide optimal medical management to limit infarct expansion until reperfusion is established.

It is also important to note that in many cases, these goals can be accomplished in parallel. Various stroke process improvement strategies can be employed to efficiently accomplish the above initial management goals. Since hospital infrastructures vary between PSCs and CSCs with established endovascular teams, the goals in this document which are specific to PSCs are separately highlighted. The SNIS ideal stroke process timelines are presented in table 1. Although we recognize that most PSCs and CSCs may not be able to meet these idealized time targets, we believe the concepts and strategies presented herein will allow PSCs (door to needle time, PSC to puncture, etc) and CSCs (door to needle time, door to puncture time, door to first recanalization, etc) to achieve these goals within 18-24 months. 
Table 1 Society of Neurointerventional Surgery suggested stroke process time metrics*

\begin{tabular}{|c|c|c|}
\hline Action & Time $(\min ) \dagger$ & SNIS 'ideal' time‡ \\
\hline Door to physician & $<10$ & On arrival \\
\hline Door to NCCT/CTA & $<25$ & On arrival \\
\hline Door to stroke team & $<15$ & $<10 \min$ \\
\hline Door to NCCT interpretation & $<45$ & $<15 \min$ \\
\hline Door to CTA interpretation & N/A & $\begin{array}{l}<20 \mathrm{~min} \\
\text { (or } 10 \mathrm{~min} \text { after acquisition) }\end{array}$ \\
\hline Door to IV tPA & $<60$ & $<30 \min$ \\
\hline Door to CTP/MRI (optional) & N/A & $<30 \min$ \\
\hline CSC Door to puncture & N/A & $<60 \min$ \\
\hline CSC Door to recanalization & $\mathrm{N} / \mathrm{A}$ & $<90 \min$ \\
\hline PSC picture to CSC puncture§ & $\mathrm{N} / \mathrm{A}$ & $<90 \min$ \\
\hline \multicolumn{3}{|c|}{$\begin{array}{l}\text { *Assuming emergency medical services prenotification. } \\
\text { †AHA } 2013 \text { standard. } \\
\text { \#SNIS ideal. } \\
\text { \$Assuming direct transfer to biplane neuroangiography suite when feasible. } \\
\text { AHA, American Heart Association; CTA, CT angiography; CTP, CT perfusion; CSC, } \\
\text { Comprehensive Stroke Center; NCCT, non-contrast CT scan; PSC, Primary Stroke } \\
\text { Center; SNIS, Society of Neurolnterventional Surgery; TPA, tissue plasminogen } \\
\text { activator. }\end{array}$} \\
\hline
\end{tabular}

\section{Stroke team activation}

Stroke code team activation ('brain attack' team of stroke nursing coordinators, emergency room physician, stroke neurologist, radiologist, CT and/or MR technologists) should be standardized independently of the mechanism by which a patient with AIS presents (emergency medical services, EMS) transport, emergency department walk-in, or an in-hospital stroke). However, specific processes will be truncated and tailored if a patient is first managed at a PSC rather than a CSC. A dedicated AIS or 'brain attack' (or any chosen name) response team should always be available to implement the parallel processing required to optimize care for these patients, rapidly accomplishing the five initial assessment/management goals. At a minimum, this activation should occur for all patients with suspected AIS presenting within $6 \mathrm{~h}$ of the time the patient was last seen to be well.

Recommendation: Early non-contrast CT (NCCT) imaging and final interpretation (ideally, $<15 \mathrm{~min}$ from presentation to PSC or CSC) is essential as further stroke care delivery is dependent on the results of this examination (AHA Class I, Level of Evidence A).

Therefore, nothing apart from hemodynamic instability or airway concerns should delay transport to the CT scanner. Ideally, patients should be transported directly from the EMS arrival entrance to the CT scanner whenever possible. Thus, EMS prenotification to alert the team and ensure the CT scanner is ready is strongly recommended. Supplemental oxygen administration and blood pressure measurements are standard of care considerations but, again, should not delay CT transport.

The in-house CT technologist must be an integral member of the 'brain attack' response team so that the CT room can be vacated and prepared for the arrival of the patient with AIS. In parallel, obtaining large-bore IV access should be of utmost importance for blood samples, iodinated contrast for CT angiography (CTA) imaging, and most importantly, IV tPA bolus/infusion. However, delays in obtaining IV access should not delay obtaining the NCCT scan. Early response of the anesthesia service may help to expedite processes, especially in situations of patient non-compliance, airway management, hemodynamic lability, vascular access, and preparation for advanced imaging and endovascular intervention.

The next two major steps are the administration of IV tPA to eligible patients and the evaluation of potential large vessel occlusion (LVO) using non-invasive imaging, predominantly CTA. Centers will have to customize workflow, but in general, these two steps should occur in parallel, as quickly as possible, and with the understanding that CTA should not delay administration of IV tPA. Examples of potential workflows are as follows:

1. At some centers, routine CTA is part of their workflow for all patients with suspected AIS.

2. For centers where CTA is not part of routine imaging in all patients with AIS, the local institution should determine a clinical severity threshold. This may be the National Institute of Health Stroke Scale (NIHSS) or other severity scale (Cincinnati Pre-hospital Stroke Scale (CPSS), ${ }^{10}$ Face, Arm, Speech Test (FAST), ${ }^{11}$ Los Angeles Motor Score (LAMS), ${ }^{12}{ }^{13}$ Rapid Arterial oCclusion Evaluation (RACE), ${ }^{14}$ Recognition of Stroke in the Emergency Room (ROSIER), ${ }^{15}$ etc). Patients meeting a prespecified threshold should have both NCCT and CTA performed upon first trip to the CT scanner.

3. In other centers where IV tPA can be initiated in the CT scanner (recommended), it would be reasonable to use the CT scanner room as a stroke treatment suite. In this workflow, IV tPA can be initiated before CTA, in the CT scanner.

The common thread in all of these workflows is that the patient does not repeatedly go back to the scanner. These workflows can be applied to both PSCs and CSCs.

If there is concern that the prothrombin time/international normalized ratio, either point of care interventional neuroradiology testing can be performed in the CT suite (where allowed) or in the laboratory where $<20$ min turnaround times should be standard (note that these tests are ineffective for new oral anticoagulants (direct thrombin or factor Xa inhibitors)). Otherwise, no blood sample data other than the finger stick blood glucose are required to make a decision as to whether to administer IV tPA.

\section{NCCT imaging}

NCCT is an excellent means of establishing if a patient with AIS is a candidate for IV tPA, as it can accurately identify hemorrhage, large areas of infarction with edema, or other findings such as large mass lesions, which would preclude IV tPA administration. ${ }^{16-19}$ However, NCCT is suboptimal for the identification of LVOs-a prerequisite to EVT for ELVO. Although the hyperdense middle cerebral artery sign can indicate $\mathrm{LVO},{ }^{20}$ it may not be sufficiently sensitive to reliably identify patients with ELVO, even with thinner slice acquisition and process optimization. ${ }^{21}$ In addition, NCCT is not as sensitive in the identification of extremely early ischemic change, and this also has implications before EVT, as will be discussed below. Despite efforts to improve NCCT sensitivity for the detection of early ischemic change with post-processing of the acquired data (windowing and leveling) ${ }^{22}$ and structured scoring systems such as the Alberta Stroke Program Early CT Score (ASPECTS), ${ }^{23}$ NCCT has overall weak sensitivity and interobserver agreement for detecting early ischemic change. ${ }^{24} 25$ Nevertheless, NCCT remains the first-line imaging in these patients, primarily because of its ability to quickly exclude hemorrhage and ASPECTS extremes (really good scores and really bad scores, particularly in combination with the CTA collateral score (see below)), and is probably adequate for most clinical decision-making. 


\section{IV tPA}

Based on the results of the two-part National Institute of Neurological Disorders and Stroke rtPA Stroke Trials, ${ }^{16}$ IV tPA $(0.9 \mathrm{mg} / \mathrm{kg}$, maximum dose $90 \mathrm{mg}$ ) was approved by the Food and Drug Administration (FDA) for use in patients with AIS presenting within $3 \mathrm{~h}$ of symptom onset. Subsequent trials (European Cooperative Acute Stroke Study (ECASS) I, ECASS II, Alteplase Thrombolysis for Acute Noninterventional Therapy in Ischemic Stroke (ATLANTIS) A, ATLANTIS B) confirmed the benefit of thrombolytic therapy for patients with AIS and time-to treatment interactions in individual and pooled analyses. $^{6}{ }^{26-29}$ While some studies report that IV tPA is beneficial up to $6 \mathrm{~h}$ from stroke onset, it is only FDA approved within $3 \mathrm{~h}$, but may be administered up to $4.5 \mathrm{~h}$ after the onset of symptoms based on the results of the ECASS III trial. ${ }^{30}$

Recommendation: Endovascular therapy should complement and not replace IV administration of recombinant $t P A$ in eligible patients (AHA Class I, Level of Evidence A).

\section{Acute non-invasive vascular imaging (CTA)}

ELVO is an acute vascular emergency (just like pulmonary embolism and aortic dissection) that is often clinically devastating $^{31} 32$ and can predict failure of IV tPA according to its location. ${ }^{33}$ Identification of LVO in association with moderate to severe acute stroke should prompt consideration of emergency EVT. $^{1-3} 34$ CTA, widely available at most hospitals, is a rapid and reliable method of identifying or excluding LVO in patients with AIS.

Clinical studies had erroneously suggested that NIHSS thresholds were an adequate surrogate marker to identify patients with ELVO. However, these early studies might have been subject to selection bias and the limited availability of emergent non-invasive brain vascular imaging. In recent clinical studies, where non-invasive vascular imaging (mostly CTA) was performed routinely in patients with AIS, we have

learned the following:

- ELVO is more common than we thought (table 2);

- Arbitrary NIHSS cut-off points are too inaccurate to identify ELVO;

- NIHSS accuracy depends on time from onset and LVO location.

Using a NIHSS cut-off value of 10, more than half of ELVOs would have been missed in the study by Maas et $a l^{35}$ (48\% sensitivity). Heldner $e t a^{37}$ found that a NIHSS cut-off value of 10 would have missed $30 \%$ of anterior circulation strokes and $60 \%$ of posterior circulation strokes and that accuracy is reduced at more delayed time points. Despite the inaccuracy of NIHSS in predicting ELVO, it has a role as a patient selection mechanism for endovascular stroke intervention as very low NIHSS with an ELVO may not require endovascular reperfusion therapy. The majority of endovascular trials specified a minimum NIHSS for enrollment (IMS-3, ${ }^{38}$ MR CLEAN, ${ }^{1}$ ESCAPE, ${ }^{2}$ REVASCAT $^{4}$ SWIFT PRIME ${ }^{5}$ ), and randomized populations with significantly elevated mean NIHSS $>10$. Confirmation of LVO in

Table 2 Studies evaluating the presence of large vessel occlusions in consecutive patients with acute ischemic stroke

\begin{tabular}{llll}
\hline Series & Year published & Sample size(N) & Incidence of LVO $\mathbf{n}(\%)$ \\
\hline Maas et $a l^{35}$ & 2009 & 699 & $377(54)$ \\
Hansen $e t ~ a \beta^{36}$ & 2014 & 637 & $183(29)$ \\
Heldner $e t a l^{37}$ & 2013 & 2152 & $1043(48)$ \\
\hline
\end{tabular}

patients with low NIHSS, has important clinical implications, ${ }^{39}$ although optimal management of patients (either with EVT or medically) remains unknown.

On modern helical CT scanners, a CTA scan from the aortic arch to the cranial vertex can be performed rapidly, usually in $15-20$ s. It has high diagnostic yield $(>50 \%)^{40}$ and the highest accuracy of any non-invasive imaging study for AIS and LVO. ${ }^{41}$ Combined head and neck scanning allows assessment of the aortic arch, carotid bifurcations, superimposed cervical pathology (proximal internal carotid artery (ICA) ruptured plaques/ occlusion or cervical dissections), as well as excellent vascular resolution of the embolus location, length, circle of Willis, and distal collaterals. Some may use the detailed anatomic evaluation for EVT planning, including considerations such as balloon guide catheter placement, proximal aspiration catheter selection, and stent retriever deployment zones. The need for any treatment of a proximal cervical carotid stenosis or occlusion can also be assessed at this time. Although the patient's renal function may remain unknown on presentation with AIS, NCCT with CTA may still be performed without prior determination of renal function in the case of a clinically devastating stroke.

Recommendation: The risk of iodinated contrast nephrotoxicity should never delay CTA to determine the presence or absence of a clinically devastating ELVO ${ }^{42}{ }^{43}$ (AHA Class I, Level of Evidence $B)$. Ideally, identification of ELVO should be completed within 10 min of CTA acquisition and the treating team informed.

MR angiography (MRA) has less sensitivity/specificity and requires more acquisition time than $\mathrm{CTA}$, it may be a suitable alternative for those with severe iodinated contrast allergies. With MRA, one can adequately confirm an ICA or M1 ELVO, for which EVT is required. In the recently published endovascular stroke intervention trials, ${ }^{1-5}$ CTA or MRA imaging was performed without significant delay during IV tPA infusion. In patients with renal insufficiency, there is no advantage in MRA of the neck as adequate imaging would also require gadolinium contrast, contraindicated owing to risks of nephrogenic systemic fibrosis.

For those centers that have abbreviated MRI protocols to optimally assess core infarct volumes for tissue-based imaging selection (see below), and cannot quickly obtain an accurate MRI screening checklist, a low-dose body tomogram (to include chest and abdomen) can also be performed at the time of NCCT/CTA to screen for a pacemaker or other foreign body that might preclude MR imaging of the patient with ELVO.

Recommendation: CTA is the most accurate and efficient noninvasive means of confirming or excluding the presence of an ELVO and should be performed as quickly as possible in those patients in whom an LVO (severe stroke) is suspected (AHA Class $I$, Level of Evidence B). Any facility that manages or receives patients with stroke (primary stroke, comprehensive stroke, or other) must have the capability to rapidly perform CTA to identify patients with ELVO. At a minimum, CTA vessel imaging should be performed in all patients who meet a predefined clinical severity threshold. MRA can be substituted for CTA in those patients with severe iodinated contrast allergy. If circumstances dictate that non-invasive vascular imaging (CTA or MRA) will unnecessarily delay EVT, it is reasonable to forgo CTA and perform catheter-directed digital subtraction angiography in conjunction with EVT as rapidly as possible.

\section{ELVO team activation}

Stroke code activation of the 'ELVO' team (neurointerventional surgeon, interventional technologist, nurse, and in some cases the anesthesiologist) is usually separate from activation of the 
medical, or non-interventional, 'brain attack' response team and will require separate, tiered activation. The criteria for activation of the interventional team can be either a clinical threshold (such as NIHSS, or other severity scale) in the prehospital or hospital setting, or based on confirmation of ELVO by CTA. Any additional advanced imaging modalities employed for tissue and/or EVT selection (multiphase CTA, CT perfusion (CTP) or MR diffusion-weighted imaging- perfusion-weighted imaging (DWI-PWI)), are usually at the discretion of the ELVO team, and should not delay team activation. The role of these additional imaging modalities is discussed below.

For patients evaluated at PSCs or other hospitals not performing EVT, transfer to a CSC as soon as possible is recommended. This call can, as with ELVO team activation, be based on either a clinical severity threshold (such as the full NIHSS, or abbreviated clinical scores such as CPSS, FAST, LAMS, RACE, ROSIER), or confirmation of LVO by CTA. Each PSC will need to develop a rapid mechanism of identifying these patients. It is generally appropriate to transfer to the closest CSC in order to achieve the greatest maximal benefit. Transfer protocols should be jointly developed by the PSC and CSCs involved, and preferably rehearsed in advance. Ideally, the patient should be ready to be transported as soon as IV tPA bolus has been administered, and as the infusion is starting. The exact method of transport as well as timing of CSC notification will have to be individualized to each PSC-CSC pair, but the goal should always remain the same -access to EVT as fast and safely as possible. In some cases, it may be necessary to mobilize transfer resources based on clinical severity threshold, before definitive confirmation of ELVO.

Informed consent for embolectomy can be obtained by any participating physician. We recommend that the family is provided with a brief summary of the published embolectomy trials, in order to facilitate the consent process. If the family is not available and the patient is not able to give consent, it may be reasonable to proceed with embolectomy in specific clinical situations where it has been established as the standard of care. Generally, this is in patients with a moderate to severe clinical deficit (such as those with an NIHSS score of $\geq 6$ ), documented occlusion of the M1 segment of the middle cerebral artery, with or without concomitant occlusion of the intracranial ICA, lack of large completed infarction by imaging, and where treatment can occur within $6 \mathrm{~h}$ from symptom onset. These criteria may further evolve as additional studies are published, and each center should have their own criteria for cases where informed consent need not be performed.

Recommendation: Independently of a patient's candidacy for IV tPA, once an ELVO is suspected either by prehospital triage or initial evaluation using a clinical scoring system and/or confirmed by CTA, the patient should be efficiently transported by the brain attack and ELVO response teams to the angiography suite, with groin puncture times ideally $<60 \mathrm{~min}$ from arrival. For patients with ELVO evaluated at a PSC, transfer to a CSC should be initiated if groin puncture can be achieved within $6 \mathrm{~h}$ of symptom onset (AHA Class 1, Level of Evidence A). Beyond $6 \mathrm{~h}$, the benefit of embolectomy is less certain (AHA Class $2 b$, Level of Evidence C).

\section{Advanced imaging of the infarct core (CTA collateral score/ CTP/MR DWI)}

Once vascular imaging has confirmed an ELVO, additional advanced imaging may be useful to ensure the patient is a candidate for embolectomy. Only the MR CLEAN trial entirely deferred screening patients for EVT with advanced imaging, possibly contributing to the lower overall clinical outcomes in their population in comparison with ESCAPE, EXTEND-IA, and SWIFT PRIME (although time to recanalization, and the modified TICI $2 b / 3$ rates achieved also contributed). The goal of imaging-based tissue selection is to identify patients who may benefit from endovascular reperfusion therapy and exclude patients who might be subjected to futile or potentially harmful recanalization. Accurately determining the infarct core has important implications for EVT, and also for the treatment of patients outside established time windows (ie, beyond $6 \mathrm{~h}$ from the time the patient was last seen to be well). Indeed, there is probably a maximal admission lesion volume compatible with favorable outcome (MALCOM) or infarct for which EVT would be futile (futile infarct). Final infarct volumes are a stronger predictor of outcome than recanalization, ${ }^{44} 45$ and this volume threshold may vary with age. ${ }^{46}$ As a standard NCCT interpretation alone is a poor predictor of infarct core (see above) and infarct growth rates are highly variable, ${ }^{47} 48$ time from onset thresholds for selecting patients with ELVO for EVT may not be an appropriate selection criterion and be shown to be insufficient and too simplistic.

The most sensitive and specific technique for detecting early infarction and the infarct core is MR DWI, ${ }^{49-51}$ even when employing structured reporting. ${ }^{24}$ Infarct core volumes that are $>70-100 \mathrm{~mL}$ before embolectomy may prohibit a clinical benefit from reperfusion, ${ }^{52-54}$ and more recent data suggest that MALCOM may be lower and may vary by age. For example, Ribo et $\mathrm{al}^{55}$ presented data showing that only $12 \%$ of patients with admission infarcts $>39 \mathrm{~mL}$ achieved a favorable outcome (modified Rankin Scale 0-2) and that octogenarians had a threshold of $15 \mathrm{~mL}$. Whereas MRI may accurately disqualify patients for embolectomy and possibly improve outcomes, ${ }^{56}$ the use of DWI to select patients for embolectomy is not widely available or practical at many institutions, is controversial, and has not been validated in a clinical trial. In addition, any infarct volume threshold and outcome data associated with these imaging methods may be a function of the speed and quality of the recanalization. For these reasons, NCCT and CTA are most commonly used and provide most data. The CTA collateral score $^{5758}$ seems to be useful and may improve the usefulness of CT in comparison with DWI.

The CTA collateral score uses information obtained during the initial CTA acquisition, and in some cases supplemented by additional phases of acquisition (multiphase CTA (mCTA)), to estimate stroke volume. The CTA collateral score, has been compared with DWI-MRI, ${ }^{59-61}$ and was used as an imaging criterion in the ESCAPE trial. ${ }^{3}$ Lack of collateral filling beyond the occluded territory (collateral score of 0 ) is strongly predictive of a DWI lesion of $\geq 100 \mathrm{~mL}$. ${ }^{60}$ Similarly, in another study, poor collateral patterns strongly correlated with a larger admission DWI lesion, and recanalization did not prevent or attenuate infarct growth. ${ }^{59}$ However, caution must be exercised in a single-phase CTA technique as the infarct core may be overestimated with incorrect (early arterial) acquisition techniques. ${ }^{62}$ To overcome these limitations and improve the physiologic selection of patients for embolectomy, the Calgary group developed a mCTA protocol to triage patients with AIS and ELVO. ${ }^{63}$ To eliminate any chance of overestimating the core by an early acquisition or poor contrast bolus, the Calgary CTA protocol generates time-resolved images from skull base to vertex in three phases (early, equilibrium, and late) separated by $8 \mathrm{~s}$. To assess the accuracy of this technique, correlation of the mCTA collateral scoring method with DWI lesion volume would require additional comparative trials, ideally a trial comparing collateral score alone against DWI-MRI for patient selection. 
The collateral score on mCTA correlates well with the ASPECTS score on NCCT and can be used to increase diagnostic accuracy and confidence of the NCCT interpretation. In addition, mCTA scans take $<20 \mathrm{~s}$ to acquire, require no additional contrast despite a marginally increased radiation dose, are relatively insensitive to patient motion, and require no complex post-processing. ${ }^{63}$ Finally, mCTA can be performed on any modern CT scanner.

\section{Bayesian decision-making}

For any individual patient, the physician's decision to perform EVT is dichotomous: either proceed to angiography or not. Any delay in making this decision, creates a fait accompli: time is brain-the greater the amount of time required to reach the decision, the lesser is the likelihood of a good outcome. According to Khatri et al, ${ }^{64}$ the chance of a good clinical outcome decreases $10 \%$ for every $30 \mathrm{~min}$ delay in revascularization.

The Bayesian approach to decision-making is helpful for the clinician. A 45-year-old with an NIHSS of 17, M1 occlusion, $50 \mathrm{~min}$ from onset is likely to benefit from successful endovascular thrombectomy irrespective of the details of the rest of the imaging. On the other hand an 86-year-old with an NIHSS of 17 with an M1 occlusion and pre-existing cognitive impairment, $300 \mathrm{~min}$ from stroke onset with severe arterial tortuosity in the arch and neck may have more limited benefit but greater risk from EVT irrespective of findings on brain imaging.

Data show that the key factors for decision-making in patients with ELVO are premorbid neurological status, age, efficiency of the interventional team (can TICI $2 \mathrm{~b} / 3$ revascularization be achieved within $90 \mathrm{~min}$ ), and an approximation of core infarct size $(<70-100 \mathrm{~mL})$. Thus, a combination of ASPECTS on NCCT together with collateral assessment on CTA (single or multi-phase) may be sufficient for making a decision about EVT (and the decision can be taken in a shorter period of time).

Recent trials show a significant treatment effect, and in some cases the effect size is large. Patients with AIS at the margins of the experimental inclusion/exclusion criteria would probably still benefit from revascularization. In the future, with further improvement in techniques, technology, and workflow, additional gains in safety, efficiency, and improvement in revascularization rates should be achieved. Extrapolation from trial data to clinical practice may cause physicians to proceed with EVT in patients who do not strictly meet trial inclusion criteria but might still benefit from treatment.

\section{Advanced imaging for tissue at risk/penumbra (CTP/MR-PWI)} Ischemic penumbra defines oligemic tissue at risk for infarction if rapid revascularization is not achieved. In all of the above discussions, the concept of penumbra is evaluated using a combination of the severity of the clinical deficit and location of the LVO. This concept is the 'clinical penumbra', or 'clinical-diffusion mismatch'. ${ }^{65}$ When this approach is used, no additional physiologic imaging is used to categorize the volume of tissue at risk. However, a variety of physiologic imaging tools aim to quantify a mismatch between volume of irreversibly damaged tissue (infarct core) and tissue at risk (penumbra). These techniques rely predominantly on perfusion imaging techniques using either dynamic CT- or MR-based imaging after injection of appropriate contrast medium.

The goal of CTP/MR DWI-PWI-based physiologic selection for endovascular stroke intervention is to detect infarct core, and also to physiologically select patients with a mismatch of potentially salvageable ('at-risk') ischemic tissue (penumbra, Tmax/mean transit time/time to peak) and non-salvageable infarcted tissue (core infarct, relative cerebral blood volume/ relative cerebral blood flow or MR-DWI). Several clinical trials have used minimum absolute mismatch ratios as an inclusion criterion (MR RESCUE, EXTEND-IA, and SWIFT PRIME) for intervention. However, various post-processing software applications and vendors have different methodologies for calculating core and penumbral volumes from CTP or MR-PWI data. ${ }^{66}$ Standardization of post-processing techniques and definitions for core infarction and penumbral volumes led to the development of the RAPID (iSchemaView, Menlo Park) software used in the DEFUSE-2 study, EXTEND-IA, and SWIFT-PRIME trials. $^{2} 552$ Despite these standardized post-processing techniques, other confounders may still be unaccounted for such as accurate selection of the arterial input function, contrast delay and dispersion effects from collaterals, and non-linearity of dynamic susceptibility contrast MR-PWI. CT perfusion is at a greater disadvantage, since both core infarct and penumbral volumes depend on post-processing calculations, as opposed to MR-DWI, which preserves a sensitive physiological marker of irreversible infarction (restricted diffusion of water in infarcted tissue). Owing to these drawbacks of perfusion imaging (CTP or MR-PWI), some have postulated that a surrogate for the penumbra may simply be reflected by the patient's clinical examination or NIHSS. ${ }^{65}$ At this time, the clinical penumbra may be the best indicator to select patients for embolectomy as long as an accurate and small core infarct estimate is available.

A study comparing clinical penumbra (NIHSS/DWI core or even NIHSS/CTA collateral score mismatch) with CTP and/or PWI is needed to resolve the perfusion imaging utility debate and time delay concerns of advanced imaging. ${ }^{67}$ Until such perfusion processing algorithms are standardized, validated, volumetric, fully automated, and do not cause any delays in reperfusion, these techniques cannot be recommended. Furthermore, with the MR CLEAN trial demonstrating efficacy of EVT in a broad range of patients without tissue-based or advanced imaging selection, a comparative trial is warranted to study if patients who are excluded by CTP or MR-DWI-PWI selection criteria would inevitably be subjected to futile or even harmful reperfusion with EVT.

Future designs of these trials must also consider the time taken to select a patient as a suitable candidate for embolectomy - the imaging we use to assess a patient with ELVO presenting within $2 \mathrm{~h}$ vs $10 \mathrm{~h}$ after symptom onset may be different. Perhaps perfusion imaging is suitable for patients with ELVO and a low NIHSS score, stratifying interventional management based on matched versus mismatch patterns. Additionally, wake up or unknown time window strokes will be more dependent on advanced imaging techniques such as CTP or MR-DWIPWI. The time frame for treatment and the optimal advanced imaging technique, if any, for embolectomy patients are intriguing factors requiring research.

Advanced imaging techniques such as multiphase CTA, CTP, or MR-DWI-PWI may offer benefits of patient and tissue selection for successful endovascular reperfusion therapy, but no consensus has been established.

Infarct core assessment can be accomplished with a variety of CT/CTA or MR-DWI-based techniques (CT-ASPECTS, CTA collateral score, DWI-ASPECTS, DWI volume). Conversely, penumbral imaging has not been validated and, as a minimum, requires standardized post-processing techniques for interpretation. Furthermore, a clinical penumbra (NIHSS) may serve as a gross, but adequate surrogate for penumbral tissue. 


\section{Upon arrival at the angiography suite}

Parallel workflow upon arrival at the biplane neuroangiography suite is also imperative. ${ }^{68}$ The ELVO team should have specific role assignments when they arrive. In addition, a standardized stroke kit (BRISK: brisk recanalization ischemic stroke kit) (tubing, drapes, syringes, catheters, devices, etc) that contains all equipment necessary for the case may be useful. Alternatively, an accessory kit can be created which quickly customizes the known standard angiography kit for an embolectomy case. Team members will have specific role assignments, but all members should be familiar with all duties so that critical functions are duplicated. Other "safe short cuts" may include not shaving the groin and delaying Foley catheter placement until after blood flow restoration. ${ }^{68}$

Although the use of general anesthesia (GA) for embolectomy may delay the start of treatment, advocates feel that the safety, quality, and speed of the procedure are improved. Conversely, operators using conscious sedation or monitored anesthesia care (MAC) protocols believe this allows for earlier treatment, less hemodynamic fluctuation, and neurological assessment of the patient throughout the case, despite concerns that patient movement could result in an increased incidence of vessel dissection or wire perforation. Converging evidence appears to favor the latter approach for patients with ELVO undergoing embolectomy. ${ }^{69-74}$ GA was actively discouraged in the ESCAPE trial and was used in $<10 \%$ of patients, suggesting that most patients can be treated with conscious sedation.

Data presented by the MR CLEAN investigators at the 2015 International Stroke Conference offer further insight into potential effects of using GA during endovascular stroke procedures. $^{75}$ Of 216 patients who entered the neuroangiography suite, 79 patients had embolectomy performed under GA and 137 under MAC with $6(4.4 \%)$ of the latter group converting to general endotracheal anesthesia (GETA) during the case. Although there was no significant difference in time to revascularization between the two groups, there was a difference in time to treatment initiation (the MAC group was 28 min faster). More importantly, in an analysis adjusted for age, NIHSS, time from onset, previous stroke, diabetes, atrial fibrillation, and ICA terminus occlusion, the beneficial treatment effect was lost in the GETA group (common adjusted $\mathrm{OR}=1.09$ (0.69 to 1.71) and found only in the MAC group (common adjusted $\mathrm{OR}=2.13$ (1.46 to 3.11$)$.

Recommendation: Embolectomy procedures should be performed with conscious sedation or MAC whenever possible. GETA should be reserved for patients who are not considered able to protect their airways for the procedure while supine or who are too uncooperative for the procedure to be performed safely (AHA Class IIb, Level of Evidence C).

\section{Prehospital ELVO management}

This topic will be the subject of a separate article. Prehospital triage of the patient with ELVO to an appropriate stroke center is critical for timely therapy. For ELVO suspected by the EMS (particularly if this suspicion is based on a clinical scoring system such as the NIHSS, LAMS, CPSS, RACE, ROSIER) during transport to hospital, SNIS advocates that EMS transports the patient directly to a CSC. Early identification of severe stroke can save hours if the patient with ELVO is taken to a CSC or other endovascular-capable stroke treatment center.

Although there appears to be a treatment effect in the 'drip and ship' paradigm, ${ }^{1} 35$ it is less robust, and IMS-3 data supported significant time savings (without IV tPA opportunity cost) when patients go directly to a CSC. ${ }^{76}$ If a patient is taken to a PSC, the EMS team should consider waiting for an ELVO to be excluded (by CTA) before completing the transport. If an ELVO is confirmed, immediate transport of the patient to a CSC is required. EMS teams should make every effort to prenotify all receiving hospitals with time last known well, family contact information, blood pressure, history of anticoagulant use, estimated time of arrival, and stroke severity using either (LAMS, RACE, or other agreed assessment tool).

Widespread deployment of mobile stroke treatment units (MSTUs) might potentially reduce times to embolectomy for ELVO via earlier activation of the ELVO team in the field and bypassing the emergency department altogether. The MSTU could potentially eliminate the need for in-house imaging, laboratories, and IV tPA administration; allow for earlier ELVO team activation and angiosuite preparation; and even identify/ confirm large artery occlusion (by on-board CTA capability). Indeed, until such time as MSTUs proliferate throughout the country the wisdom of the Helsinki group should not go unnoticed, "The key to success in reducing delays is to do only the basics when the patient has arrived, and to do as much as possible before and during transport."

Recommendation: A patient in the field with a suspected ELVO by EMS (based on an appropriate field severity score) should be triaged to the closest CSC, bypassing other facilities as patient stability, local policy, and additional transport time (geography) allow (AHA Class I, Level of Evidence A).

\section{Summary}

There is now the highest level of medical evidence (AHA Class I, Level of Evidence A) that embolectomy using the latest technology is the standard of care for patients with ELVO, with or without an IV tPA bridge. Any hospital that receives patients with stroke must have highly coordinated systems of care to deliver fast and efficient door to picture, needle, and puncture times all while providing the best medical management until reperfusion is achieved. To improve the provision of care, processes (delivery innovation) should be iterative and designed so that teams can cycle back, evaluate their performance, and drive process improvement for the benefit of all patients with ELVO. Finally, this committee believes that prospective data reporting (such as that provided by the SNIS neurovascular quality initiative) should be carried out for all EVT procedures for stroke.

\footnotetext{
Author affiliations

${ }^{1}$ Warren Alpert Medical School of Brown University, Providence, Rhode Island, USA ${ }^{2}$ Northwestern University Feinberg School of Medicine, Chicago, Illinois, USA ${ }^{3}$ Department of Neurosurgery, University of Calgary, Calgary, Alberta, Canada ${ }^{4}$ Department of Neurosurgery, University of Cincinnati, Cincinnati, Ohio, USA ${ }^{5}$ Department of Neurointerventional Surgery, Christiana Care Health Systems, Newark, Delaware, USA

${ }^{6}$ Department of Neurosurgery, Semmes-Murphey Clinic, Memphis, Tennessee, USA ${ }^{7}$ Department of Neurosurgery, Cedars-Sinai Medical Center, Los Angeles, California, USA

${ }^{8}$ Department of Neurological Surgery, Barrow Neurological Institute, Phoenix, Arizona, USA

${ }^{9}$ Department of Radiology, Erlanger Medical Center, Chattanooga, Tennessee, USA

${ }^{10}$ Department of Neurosurgery, Yale University School of Medicine, New Haven, Connecticut, USA

${ }^{11}$ Department of Neurological Sciences, Rush University Medical Center, Chicago, Illinois, USA

${ }^{12}$ Department of Interventional Neuroradiology, Abbott Northwestern Hospital, Minneapolis, Minnesota, USA

${ }^{13}$ Department of Neurological Surgery, University of Kentucky, Lexington, Kentucky, USA

${ }^{14}$ Radiology Imaging Associates, Interventional Neuroradiology, Englewood, Colorado, USA
} 
${ }^{15}$ Department of Neurological Surgery, Rutgers University—New Jersey Medical School, University of Medicine and Dentistry of New Jersey, Newark, New Jersey, USA

${ }^{16}$ Department of Radiology, Forsyth Medical Center, Winston Salem, North Carolina, USA

${ }^{17}$ Department of Radiology, UCSF, San Francisco, California, USA

${ }^{18}$ Cerebrovascular Center, Cleveland Clinic, Cleveland, Ohio, USA

${ }^{19}$ Department of Neurosurgery, Royal University Hospital, University of

Saskatchewan, Saskatoon, Saskatchewan, Canada

${ }^{20}$ Department of Interventional Neuroradiology, Houston Methodist Neurological

Institute, Houston Methodist Hospital, Houston, Texas, USA

${ }^{21}$ Department of Radiology, The University of Chicago, Chicago, Illinois, USA

${ }^{22}$ Department of Neurointerventional Surgery, Massachusetts General Hospital, Boston, Massachusetts, USA

${ }^{23}$ Department of Neurointerventional Surgery, Columbia Presbyterian Hospital, New York, New York, USA

${ }^{24}$ Department of Neuroradiology, UT Southwestern, Dallas, Texas, USA

${ }^{25}$ Department of Neurological Surgery, New York Presbyterian Hospital, Weill Cornell Medical College, New York, New York, USA

${ }^{26}$ Department of Neurosurgery, University of Virginia, Charlottesville, Virginia, USA

${ }^{27}$ Department of Radiology, Banner Good Samaritan Medical Center, Phoenix,

Arizona, USA

Competing interests None declared.

Provenance and peer review Commissioned; internally peer reviewed.

\section{REFERENCES}

1 Berkhemer OA, Fransen PS, Beumer D, et al. A randomized trial of intraarterial treatment for acute ischemic stroke. N Engl J Med 2015;372:11-20.

2 Campbell BC, Mitchell PJ, Kleinig TJ, et al. Endovascular therapy for ischemic stroke with perfusion-imaging selection. N Engl J Med 2015;372:1009-18.

3 Goyal M, Demchuk AM, Menon BK, et al. Randomized assessment of rapid endovascular treatment of ischemic stroke. N Engl J Med 2015;372:1019-30.

4 Jovin TG, Chamorro A, Cobo E, et al. Thrombectomy within 8 hours after symptom onset in ischemic stroke. N Engl J Med 2015;372:2296-306

5 Saver JL, Goyal M, Bonafe A, et al. Stent-retriever thrombectomy after intravenous t-PA vs. t-PA alone in stroke. N Engl J Med 2015;372:2285-95.

6 Lees KR, Bluhmki $E$, von Kummer $R$, et al. Time to treatment with intravenous alteplase and outcome in stroke: an updated pooled analysis of ECASS, ATLANTIS, NINDS, and EPITHET trials. Lancet 2010;375:1695-703.

7 Vagal AS, Khatri P, Broderick JP, et al. Time to angiographic reperfusion in acute ischemic stroke: decision analysis. Stroke 2014;45:3625-30.

8 Fransen PS, For the $\mathrm{MCl}$. Time to reperfusion and effect of intra-arterial treatment in the MR. CLEAN Trial. International Stroke Conference; Nashville, TN, 2015.

9 Jayaraman MV, Hussain MS, Abruzzo T, et al. Embolectomy for stroke with emergent large vessel occlusion (ELVO): report of the Standards and Guidelines Committee of the Society of Neurolnterventional Surgery. J Neurointerv Surg 2015;7:316-21.

10 Kothari RU, Pancioli A, Liu T, et al. Cincinnati prehospital stroke scale: reproducibility and validity. Ann Emerg Med 1999;33:373-8.

11 Harbison J, Hossain 0 , Jenkinson D, et al. Diagnostic accuracy of stroke referrals from primary care, emergency room physicians, and ambulance staff using the face arm speech test. Stroke 2003:34:71-6.

12 Nazliel B, Starkman S, Liebeskind DS, et al. A brief prehospital stroke severity scale identifies ischemic stroke patients harboring persisting large arterial occlusions. Stroke 2008;39:2264-7.

13 Kidwell CS, Saver JL, Schubert GB, et al. Design and retrospective analysis of the Los Angeles Prehospital Stroke Screen (LAPSS). Prehosp Emerg Care 1998;2:267-73.

14 Perez de la Ossa N, Carrera D, Gorchs M, et al. Design and validation of a prehospital stroke scale to predict large arterial occlusion: the rapid arterial occlusion evaluation scale. Stroke 2014;45:87-91.

15 Nor AM, Davis J, Sen B, et al. The Recognition of Stroke in the Emergency Room (ROSIER) scale: development and validation of a stroke recognition instrument. Lancet Neurol 2005;4:727-34.

16 Group TNIoNDaSr-PSS. Tissue plasminogen activator for acute ischemic stroke. The National Institute of Neurological Disorders and Stroke rt-PA Stroke Study Group. N Engl J Med 1995;333:1581-7.

17 Larrue V, von Kummer R, del Zoppo G, et al. Hemorrhagic transformation in acute ischemic stroke. Potential contributing factors in the European Cooperative Acute Stroke Study. Stroke 1997;28:957-60.

18 von Kummer R, Bourquain H, Bastianello $\mathrm{S}$, et al. Early prediction of irreversible brain damage after ischemic stroke at CT. Radiology 2001;219:95-100.

19 Wahlgren N, Ahmed N, Eriksson N, et al. Multivariable analysis of outcome predictors and adjustment of main outcome results to baseline data profile in randomized controlled trials: Safe Implementation of Thrombolysis in Stroke-MOnitoring STudy (SITS-MOST). Stroke 2008;39:3316-22.

20 Moulin T, Cattin F, Crepin-Leblond T, et al. Early CT signs in acute middle cerebral artery infarction: predictive value for subsequent infarct locations and outcome. Neurology 1996;47:366-75.

21 Mair G, Boyd EV, Chappell FM, et al. Sensitivity and specificity of the hyperdense artery sign for arterial obstruction in acute ischemic stroke. Stroke 2015;46:102-7.

22 Lev MH, Farkas J, Gemmete JJ, et al. Acute stroke: improved nonenhanced CT detection-benefits of soft-copy interpretation by using variable window width and center level settings. Radiology 1999;213:150-5.

23 Barber PA, Demchuk AM, Zhang J, et al. Validity and reliability of a quantitative computed tomography score in predicting outcome of hyperacute stroke before thrombolytic therapy. ASPECTS Study Group. Alberta Stroke Programme Early CT Score. Lancet 2000;355:1670-4.

24 McTaggart RA, Jovin TG, Lansberg MG, et al. Alberta Stroke Program Early Computed Tomographic Scoring Performance in a Series of Patients Undergoing Computed Tomography and MRI: Reader Agreement, Modality Agreement, and Outcome Prediction. Stroke 2015;46:407-12.

25 Weir NU, Pexman JH, Hill MD, et al. How well does ASPECTS predict the outcome of acute stroke treated with IV tPA? Neurology 2006;67:516-18.

26 Clark WM, Albers GW, Madden KP, et al. The rtPA (alteplase) 0- to 6-hour acute stroke trial, part A (A0276 g): results of a double-blind, placebo-controlled, multicenter study. Thromblytic therapy in acute ischemic stroke study investigators. Stroke 2000;31:811-16

27 Clark WM, Wissman S, Albers GW, et al. Recombinant tissue-type plasminogen activator (Alteplase) for ischemic stroke 3 to 5 hours after symptom onset. The ATLANTIS Study: a randomized controlled trial. Alteplase Thrombolysis for Acute Noninterventional Therapy in Ischemic Stroke. JAMA 1999;282:2019-26.

28 Hacke W, Kaste M, Fieschi C, et al. Intravenous thrombolysis with recombinant tissue plasminogen activator for acute hemispheric stroke. The European Cooperative Acute Stroke Study (ECASS). JAMA 1995;274:1017-25.

29 Hacke W, Kaste M, Fieschi C, et al. Randomised double-blind placebo-controlled trial of thrombolytic therapy with intravenous alteplase in acute ischaemic stroke (ECASS II). Second European-Australasian Acute Stroke Study Investigators. Lancet 1998;352:1245-51.

30 Hacke W, Kaste M, Bluhmki E, et al. Thrombolysis with alteplase 3 to 4.5 hours after acute ischemic stroke. N Engl J Med 2008;359:1317-29.

31 Furlan A, Higashida R, Wechsler L, et al. Intra-arterial prourokinase for acute ischemic stroke. The PROACT II study: a randomized controlled trial. Prolyse in Acute Cerebral Thromboembolism. JAMA 1999;282:2003-11.

32 Smith WS, Tsao JW, Billings ME, et al. Prognostic significance of angiographically confirmed large vessel intracranial occlusion in patients presenting with acute brain ischemia. Neurocrit Care 2006:4:14-7.

33 Saqqur M, Tsivgoulis G, Molina CA, et al. Symptomatic intracerebral hemorrhage and recanalization after IV rt-PA: a multicenter study. Neurology 2008;71:1304-12.

34 Saver JL, For the S-PI. Solitaire FR with the intention for thrombectomy as primary endovascular treatment for acute ischemic stroke. International Stroke Conference 2015; Nashville TN: American Heart Association, 2015.

35 Maas MB, Furie KL, Lev MH, et al. National Institutes of Health Stroke Scale score is poorly predictive of proximal occlusion in acute cerebral ischemia. Stroke 2009;40:2988-93.

36 Hansen CK, Christensen A, Ovesen C, et al. Stroke severity and incidence of acute large vessel occlusions in patients with hyper-acute cerebral ischemia: results from a prospective cohort study based on CT-angiography (CTA). Int J Stroke 2014;10:336-42.

37 Heldner MR, Zubler C, Mattle HP, et al. National Institutes of Health stroke scale score and vessel occlusion in 2152 patients with acute ischemic stroke. Stroke 2013;44:1153-7.

38 Broderick JP, Palesch YY, Demchuk AM, et al. Endovascular therapy after intravenous t-PA versus t-PA alone for stroke. N Engl J Med 2013;368:893-903.

39 Rajajee V, Kidwell C, Starkman S, et al. Early MRI and outcomes of untreated patients with mild or improving ischemic stroke. Neurology 2006;67:980-4.

40 Deipolyi AR, Hamberg LM, Gonzalez RG, et al. Diagnostic yield of emergency department arch-to-vertex $\mathrm{CT}$ angiography in patients with suspected acute stroke. AJNR Am J Neuroradiol 2015;36:265-8.

41 Lev MH, Farkas J, Rodriguez VR, et al. CT angiography in the rapid triage of patients with hyperacute stroke to intraarterial thrombolysis: accuracy in the detection of large vessel thrombus. J Comput Assist Tomogr 2001;25:520-8.

42 Krol AL, Dzialowski I, Roy J, et al. Incidence of radiocontrast nephropathy in patients undergoing acute stroke computed tomography angiography. Stroke 2007;38:2364-6.

43 McDonald RJ, McDonald JS, Carter RE, et al. Intravenous contrast material exposure is not an independent risk factor for dialysis or mortality. Radiology 2014:273:714-25.

44 Yoo AJ, Chaudhry ZA, Nogueira RG, et al. Infarct volume is a pivotal biomarker after intra-arterial stroke therapy. Stroke 2012;43:1323-30.

45 Zaidi SF, Aghaebrahim A, Urra X, et al. Final infarct volume is a stronger predictor of outcome than recanalization in patients with proximal middle cerebral artery occlusion treated with endovascular therapy. Stroke 2012;43:3238-44. 
46 Ribo M, Flores A, Mansilla $E$, et al. Age-adjusted infarct volume threshold for good outcome after endovascular treatment. J Neurointerventional Surg 2014;6:418-22.

47 Hakimelahi R, Vachha BA, Copen WA, et al. Time and diffusion lesion size in major anterior circulation ischemic strokes. Stroke 2014;45:2936-41.

48 Wheeler HM, Mlynash M, Inoue M, et al. The growth rate of early DWI lesions is highly variable and associated with penumbral salvage and clinical outcomes following endovascular reperfusion. Int J Stroke 2015;10:723-9.

49 Barber PA, Darby DG, Desmond PM, et al. Identification of major ischemic change. Diffusion-weighted imaging versus computed tomography. Stroke 1999;30:2059-65.

50 Fiebach JB, Schellinger PD, Jansen 0 , et al. CT and diffusion-weighted MR imaging in randomized order: diffusion-weighted imaging results in higher accuracy and lower interrater variability in the diagnosis of hyperacute ischemic stroke. Stroke 2002;33:2206-10.

51 Gonzalez RG, Schaefer PW, Buonanno FS, et al. Diffusion-weighted MR imaging: diagnostic accuracy in patients imaged within 6 hours of stroke symptom onset. Radiology 1999;210:155-62.

52 Lansberg MG, Straka M, Kemp S, et al. MRI profile and response to endovascular reperfusion after stroke (DEFUSE 2): a prospective cohort study. Lancet Neurol 2012;11:860-7.

53 Mlynash M, Lansberg MG, De Silva DA, et al. Refining the definition of the malignant profile: insights from the DEFUSE-EPITHET pooled data set. Stroke 2011;42:1270-5.

54 Yoo AJ, Verduzco LA, Schaefer PW, et al. MRI-based selection for intra-arterial stroke therapy: value of pretreatment diffusion-weighted imaging lesion volume in selecting patients with acute stroke who will benefit from early recanalization. Stroke 2009;40:2046-54.

55 Ribo M, Tomasello A, Lemus $M$, et al. Maximal admission core lesion compatible with favorable outcome in acute stroke patients undergoing endovascular procedures. International Stroke Conference; Nashville, TN, 2015.

56 Wisco D, Uchino K, Saqqur M, et al. Addition of hyperacute MRI AIDS in patient selection, decreasing the use of endovascular stroke therapy. Stroke 2014;45:467-72

57 Menon BK, Smith EE, Modi J, et al. Regional leptomeningeal score on CT angiography predicts clinical and imaging outcomes in patients with acute anterior circulation occlusions. AJNR Am J Neuroradiol 2011;32:1640-5.

58 Miteff $F$, Levi CR, Bateman GA, et al. The independent predictive utility of computed tomography angiographic collateral status in acute ischaemic stroke. Brain 2009;132(Pt 8):2231-8.

59 Nambiar V, Sohn SI, Almekhlafi MA, et al. CTA collateral status and response to recanalization in patients with acute ischemic stroke. AJNR Am J Neuroradiol 2014;35:884-90.

60 Souza LC, Yoo AJ, Chaudhry ZA, et al. Malignant CTA collateral profile is highly specific for large admission DWI infarct core and poor outcome in acute stroke. AJNR Am J Neuroradiol 2012;33:1331-6.

61 Cheng-Ching E, Frontera JA, Man S, et al. Degree of collaterals and not time is the determining factor of core infarct volume within 6 hours of stroke onset. AJNR Am J Neuroradiol 2015;36:1272-6.
62 Yoo AJ, Hu R, Hakimelahi R, et al. CT angiography source images acquired with fast-acquisition protocol overestimate infarct core on diffusion weighted images in acute ischemic stroke. J Neuroimaging 2012;22:329-35.

63 Menon BK, d'Esterre CD, Qazi EM, et al. Multiphase CT angiography: a new tool for the imaging triage of patients with acute ischemic stroke. Radiology 2015:275:510-20.

64 Khatri P, Abruzzo T, Yeatts SD, et al. Good clinical outcome after ischemic stroke with successful revascularization is time-dependent. Neurology 2009;73:1066-72.

65 Boxerman JL, Jayaraman MV, Mehan WA, et al. Clinical stroke penumbra: use of National Institutes of Health stroke scale as a surrogate for CT perfusion in patient triage for intra-arterial middle cerebral artery stroke therapy. AJNR Am J Neuroradiol 2012;33:1893-900.

66 Schaefer PW, Souza L, Kamalian S, et al. Limited reliability of computed tomographic perfusion acute infarct volume measurements compared with diffusion-weighted imaging in anterior circulation stroke. Stroke 2015;46:419-24.

67 Sheth KN, Terry JB, Nogueira RG, et al. Advanced modality imaging evaluation in acute ischemic stroke may lead to delayed endovascular reperfusion therapy without improvement in clinical outcomes. J Neurointerventional Surg 2013; 5(Suppl 1):i62-5.

68 Goyal M, Menon BK, Hill MD, et al. Consistently achieving computed tomography to endovascular recanalization $<90$ minutes: solutions and innovations. Stroke 2014;45:e252-6.

69 Abou-Chebl A, Lin R, Hussain MS, et al. Conscious sedation versus general anesthesia during endovascular therapy for acute anterior circulation stroke: preliminary results from a retrospective, multicenter study. Stroke 2010;41:1175-9.

70 Davis MJ, Menon BK, Baghirzada LB, et al. Anesthetic management and outcome in patients during endovascular therapy for acute stroke. Anesthesiology 2012;116:396-405.

71 Hassan $A E$, Chaudhry SA, Zacharatos $H$, et al. Increased rate of aspiration pneumonia and poor discharge outcome among acute ischemic stroke patients following intubation for endovascular treatment. Neurocrit Care 2012;16:246-50

72 John S, Thebo U, Gomes J, et al. Intra-arterial therapy for acute ischemic stroke under general anesthesia versus monitored anesthesia care. Cerebrovasc Dis 2014;38:262-7

73 Jumaa MA, Zhang F, Ruiz-Ares G, et al. Comparison of safety and clinical and radiographic outcomes in endovascular acute stroke therapy for proximal middle cerebral artery occlusion with intubation and general anesthesia versus the nonintubated state. Stroke 2010;41:1180-4.

74 Nichols C, Carrozzella J, Yeatts $S$, et al. Is periprocedural sedation during acute stroke therapy associated with poorer functional outcomes? I Neurointerventional Surg 2010;2:67-70.

75 Berkhemer $\mathrm{OA}$, for the $\mathrm{MCl}$. Impact of general anesthesia on treatment effect in the MR. CLEAN trial. International Stroke Conference; Nashville, Tennessee, 2015.

76 Goyal M, Almekhlafi MA, Fan L, et al. Evaluation of interval times from onset to reperfusion in patients undergoing endovascular therapy in the Interventional Management of Stroke III trial. Circulation 2014;130:265-72. 